\title{
Design and causal modelling of a piezoelectric multi-actuator system used in forging processes
}

\author{
T. H. Nguyen, C. Giraud-Audine, M. Amberg, B. Lemaire-Semail, G. Abba, R. Bigot
}

\begin{abstract}
This paper presents the design of a mechatronic system integrating piezoelectric multi-actuators, which is used to generate low-frequency vibrations to assist the forging process. With the aim of controlling the complete system, modelling using Energetic Macroscopic Representation is performed. A prototype with an electrical system is developed in a short term to validate the design. Finally, the preliminary experiments are presented with the corresponding simulation's results.
\end{abstract}

Index Terms-Design, Modelling, Compliant mechanism, Energetic Macroscopic Representation, Forging, Graphical model, Piezoelectric actuator

\section{NOMENCLATURE}

$\omega_{0} \quad$ Lower die's instantaneous rotation speed

$\omega_{x} \quad$ Rotation speed of lower die around Ox

$\omega_{y} \quad$ Rotation speed of lower die around Oy

K Compliant mechanism's stiffness matrix

M Compliant mechanism's inertia matrix

$\mathbf{F}_{\mathbf{e}}$ External forces' vector

q Motional variables' vector

$\mathbf{v}_{\mathbf{0}} \quad$ velocity's vector of point $\mathrm{O}$

$\mathbf{F}_{\mathbf{0}} \quad$ Resultant force's vector of point $\mathrm{O}$

$K_{S} \quad$ Stiffness of piezoelectric actuator

$K_{C} \quad$ Electromechanical conversion factor

$C$ Piezoelectric actuator's capacitance

$U_{p} \quad$ Piezoelectric actuator's voltage

$i_{p} \quad$ Current entering the actuator

$U_{s} \quad$ Continuous supply voltage

$i_{s} \quad$ Current passing through the diode

$C_{0} \quad$ Inverter's capacitance

$U_{0} \quad$ Inverter's DC bus voltage

$[\mathrm{rad} / \mathrm{s}]$

$[\mathrm{rad} / \mathrm{s}]$

$[\mathrm{rad} / \mathrm{s}]$

$[\mathrm{N} / \mathrm{m}]$

[kg]

$[\mathrm{N}]$

[m]

$[\mathrm{m} / \mathrm{s}]$

$[\mathrm{N}]$

$[\mathrm{N} / \mathrm{m}]$

$[\mathrm{C} / \mathrm{m}]$

$[\mathrm{F}]$

[V]

[A]

[V]

[A]

$[\mathrm{F}]$

[V]

\section{INTRODUCTION}

The advantage of vibrations in forging process has been reported in different studies. By using ultrasonic vibrations, a significant reduction of force has been obtained during forging a specimen of plasticine [1], or one of aluminium [2]. The vibrations in low frequencies (from few Hertz to few hundreds Hertz), generated by piezoelectric actuators in the direction of forging motion, has been also proved to have an important influence in the reduction of forging force in the test with plasticine [3] or with a small metallic specimen [4], [5]. However, the maximal force generated by piezoelectric actuator in these studies is still limited. In order to increase the applied force, a proposed solution is to increase the number of piezoelectric actuators. Moreover, the

T. H. Nguyen, C. Giraud-Audine are with Laboratoire d'Electrotechnique et d'Electronique de Puissance de Lille (L2EP), Arts et Métiers Paristech centre de Lille, 59046 Lille, France (email: thanh-hung.nguyen@ensam.eu)

M. Amberg, B. Lemaire-Semail are with Laboratoire d'Electrotechnique et d'Electronique de Puissance de Lille (L2EP), Université Lille 1, Villeneuve d'Ascq, France

G. Abba and R. Bigot are with Laboratoire de Conception, Fabrication et Commande (LCFC), Arts et Métiers Paristech centre de Metz, Metz, France (email: gabriel.abba@ensam.eu) configuration of multi-actuator system allows us to generate more complicated vibrations than a vertical vibration in the forging's direction. A recent work with numerical simulation has shown that the forging's force can be significantly reduced due to a movement following a progressive wave of the lower die applied to the workpiece during forging process [6].

The objective of this study is to design a piezoelectric multi-actuator system in order to generate a progressive wave in the combination with a vertical vibration to assist forging process. This system includes a mechanism integrating multi-actuators and an electrical system which is used to generate the desired voltage's waveform of actuators. In the further aim of controlling this system, a model for the complete system using the Energetic Macroscopic Representation is performed in the second part of this paper. The experimental results are also presented to validate the system's function and compared to the simulated ones.

\section{MeChaniCAL SYSTEM}

\section{A. Kinematics' requirements}

Consider a frame Oxyz attached to the center $\mathrm{O}$ of the lower die's surface in contact with the workpiece as in Fig.1. A vector $\overrightarrow{\omega_{0}}(t)$ is an instantaneous rotation vector of the solid lower die around point O. The lower die's movement following a progressive wave is defined by a rotation of vector $\overrightarrow{\omega_{0}}(t)$ around $\mathrm{Oz}$ with the frequency $f$.

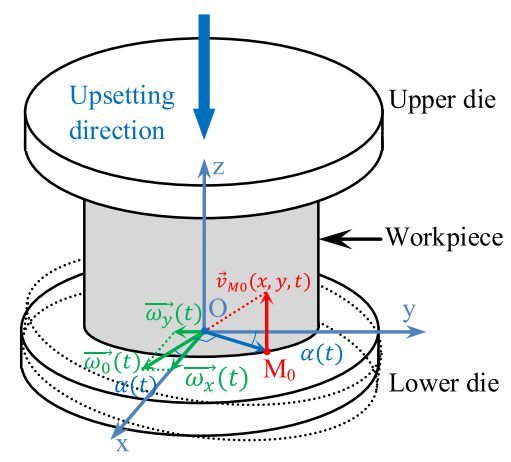

Fig. 1. Kinematic diagram of a progressive wave on the lower die

To obtain the desired motion, the projections of the vector $\overrightarrow{\omega_{0}}(t)$ into two axes Ox and Oy are defined by:

$$
\begin{aligned}
& \omega_{x}(t)=\omega_{0} \cos (2 \pi f t+\phi) \\
& \omega_{y}(t)=\omega_{0} \sin (2 \pi f t+\phi)
\end{aligned}
$$

where $\phi$ is the initial angle of rotation.

In order to obtain a progressive wave's movement of the lower die defined in (1), the mechanism must have two rotational degrees of freedom (d.o.f) around two axes Ox and Oy. Moreover, it must also allow a displacement in 
the upsetting's direction (direction $\mathrm{Oz}$ ) to generate a vertical vibration. The kinematic diagram of the desired mechanical system is presented in Fig.2.

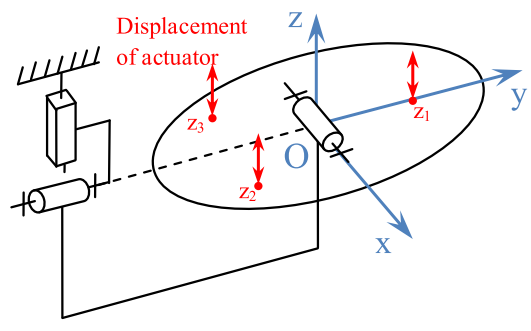

Fig. 2. Kinematic diagram of mechanical system

A balanced configuration with three multilayer piezoelectric actuators placed equidistant along a circle under the lower die is proposed to produce two rotations combined with a vertical movement. To avoid the mechanical component's tolerance, which is incompatible with the small displacement of piezoelectric actuators $(60 \mu \mathrm{m})$, a compliant mechanism is considered for the guiding of the mechanical system.

\section{B. Compliant mechanism}

This solution is largely used in the applications related to the small displacement with high precision to eliminate backlash, wear and allows a very high resolution [7]. In this application, a parallel structure of three flexible beams connected to the lower die (Fig.3) is used to have an out-ofplan movement. A displacement along $\mathrm{Oz}$ and two rotations around Ox, Oy can be achieved with the three vertical displacements at three points $I_{1}, I_{2}, I_{3}$.

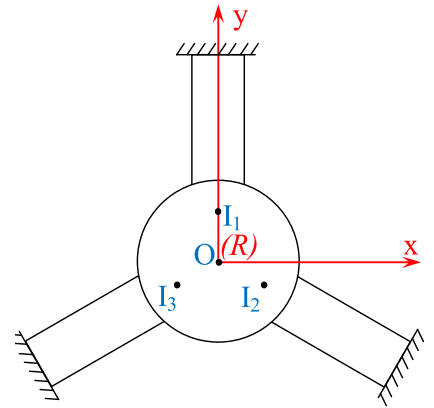

Fig. 3. Design with compliant guiding for a 3 d.o.f movement

The compliant mechanism's stiffness is expressed as a stiffness matrix along the six possible movements of lower die at the center $\mathrm{O}$. This stiffness is approximately determined by a sum of transformed stiffness of each flexible beam in the local frame $\left(R_{i}\right)$ to the point $\mathrm{O}$ of the global frame $(R)$. The compliant mechanism's stiffness is formulated as follows [8]:

$$
{ }^{R} \mathbf{K}_{O}=\sum_{i=1}^{3}{ }^{R_{i}} \mathbb{T}_{R}^{T} \cdot{ }^{R_{i}} \mathbf{K}_{O_{i}} \cdot{ }^{R_{i}} \mathbb{T}_{R}
$$

where ${ }^{R_{i}} \mathbb{T}_{R}$ the screw transformation of the frame $\left(R_{i}\right)$ to the frame $(R)$ expressed in the frame $\left(R_{i}\right)$ and $R_{i} \mathbf{K}_{O_{i}}$ is the stiffness matrix of single beam, expressed in the local frame $\left(R_{i}\right)$ ( [9], [10]). An analytic calculation presents this stiffness matrix in the following form :

$$
{ }^{R} \mathbf{K}_{O}=\left[\begin{array}{cccccc}
k_{1} & 0 & 0 & 0 & -k_{2} & 0 \\
0 & k_{1} & 0 & k_{2} & 0 & 0 \\
0 & 0 & k_{5} & 0 & 0 & 0 \\
0 & k_{2} & 0 & k_{3} & 0 & 0 \\
-k_{2} & 0 & 0 & 0 & k_{3} & 0 \\
0 & 0 & 0 & 0 & 0 & k_{4}
\end{array}\right]
$$

The system must have low stiffness in the desired movements' direction but very high stiffness in the other directions. The dimensions of the flexible beams are chosen to ensure that $k_{5} \ll k_{1}$ and $k_{3} \ll k_{4}$ and that the beam's deformation is still in the elastic domain with the maximal displacement of actuators.

\section{Mechanical coupling with actuators}

To avoid moments or forces perpendicular to the direction of movement of the actuator, punctual contacts are used in this design for their robustness and simplicity. However, the die may be separated from the contact under the effect of inertia during vibrations. A continuous compressive force between the contact and the lower die is thus required to maintain a permanent contact with the lower die. A structure, called contact carrier presented in Fig.4a, is used to maintain these permanent contacts. It also allows a bi-directional actuation thanks to the pre-stressed force in the actuator. The actuators are screwed to the contact carriers, and so are the spherical caps. The spherical caps' radius is chosen to reduce the contact's deformation and improve its stiffness. These caps are in contact with a secondary plate (Fig.4b) fixed to the lower die in order to have a clear space above the lower die for the forging's operations.

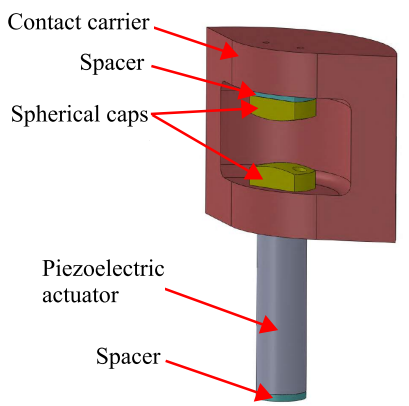

(a) Contact carrier

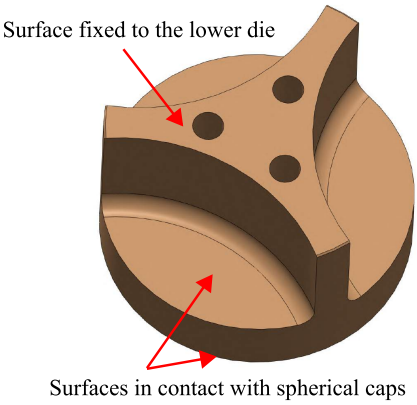

(b) Secondary plate
Fig. 4. Components for punctual contacts

A spacer will be used to apply a pre-load on the contacts during the assembly by thermal expansion of contact carrier. Finally, the dimensional dispersion of actuators will be eliminated by the use of another spacer between the actuator and the base plate (Fig.5). The calibration by spacer also helps reduce the possible stress, caused by the assembly, in the deformable beam. For the objective of controlling the lower die's movement, three eddy current sensors are used for the measurement of two rotation angles and one vertical displacement.

\section{Prototype}

A prototype of the mechanical system in PVC material is built in the first step to validate the functionalities of the design. To adapt to this kind of material and simplify 


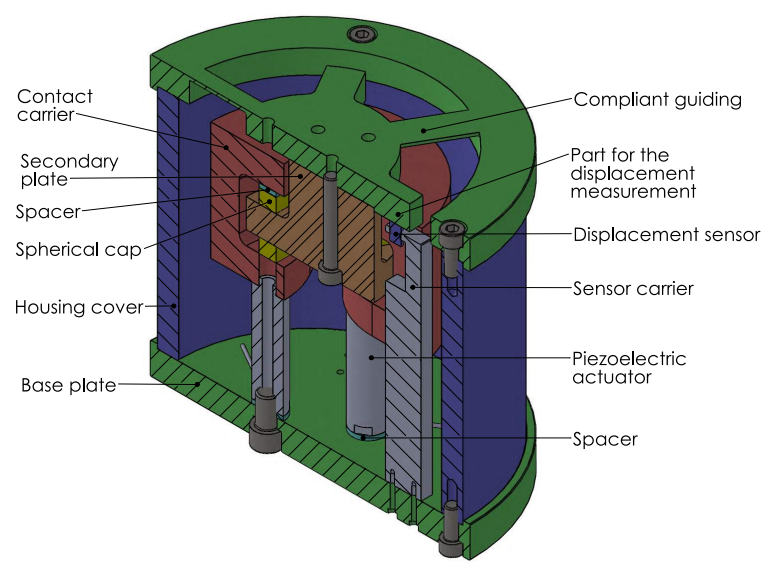

Fig. 5. Section view of the mechanical system's design

the fabrication as well as the measurement of lower die's movement, some details are changed with respect to the presented design. The punctual contact is constructed by a steel ball attached to the actuator by a plastic screw cap as shown in Fig.6a. Due to the small diameter of the steel ball, the allowed force is kept below twenty Newtons to avoid plastic deformation. To maintain a permanent contact in this prototype, magnets are used to produce a constant pre-load between the steel ball and the aluminium disc fixed with the compliant mechanism.

An aluminium plate is placed at the center of the lower die to have a rigid surface in contact with the steel balls. The housing cover is changed to triangular form to facilitate the fabrication and to reduce the overall dimension (Fig.6b). The displacement sensors are built from current sensors (Hall effect) which are fixed to the stationary part while the magnets are fixed on the lower die in the opposite position (see Fig.6a). The sensors measure the magnetic field of the magnets to estimate the lower die's displacement. The assembled system is presented in Fig.6c.

\section{ELECTRICAL SYSTEM}

To generate a progressive wave, the three piezoelectric actuators are supplied at high voltage (from $-200 \mathrm{~V}$ to $1000 \mathrm{~V}$ correspond to a maximal displacement of actuators) by a three-phase power supply. In addition, this supply system must be able to provide a variable frequency voltage in order to change the vibrations' frequency (from $5 \mathrm{~Hz}$ to $100 \mathrm{~Hz}$ ). This kind of power supply can be performed by an analog power amplifier [11], [12] or a switching power supply. Due to the limited efficiency of analog power amplifier, the switching power supply method is more largely applied to the piezoelectric actuators' power supply [13]-[16]. This method is often realised by using a transformer to increase the output voltage. However, the design with transformer becomes more complicated to obtain a bias of actuators' voltage. In this paper, for the availability and portability of actual system, an electrical system without transformer using a commercial inverter Semikron is proposed to supply a high voltage with variable frequency from a constant voltage source. The power supply's circuit diagram is presented in the Fig.7.

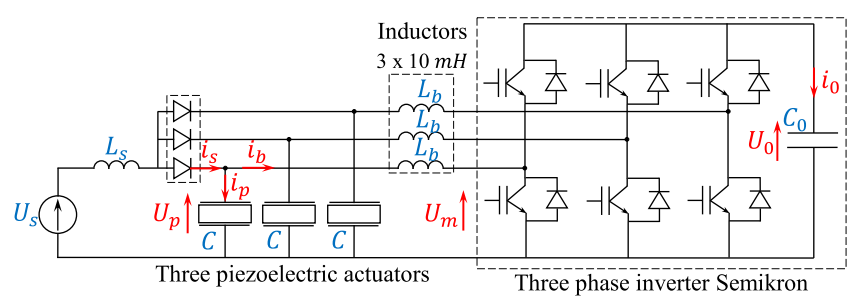

Fig. 7. Scheme for three piezoelectric actuators' power supply

The principle is to use the inverter as a voltage booster to obtain a high voltage output. The external power supply is represented by the voltage source $U_{s}$ which provides a constant voltage to the system through an inductor $L_{s}$ and diodes. In the charging period, the diodes are conducted and the capacitor $C_{0}$ is charged through three branches of inverter. In the phase of vibrations' generation, the inverter is used to convert the bus voltage of capacitor $C_{0}$ in a threephase variable frequency voltage using the PWM method. The three-phase voltage is then applied to the actuators represented by capacitors $C$. The inductors $L_{b}$ and $L_{s}$ are necessary to avoid direct connections between capacitors $C_{0}, C$ and between capacitors $C$ and voltage source $U_{s}$. The inductor $L_{b}$ is chosen so that the cut-off frequency of the filter $L_{b}-C$ is quite high, $3 \mathrm{kHz}$ so compared to the waveforms' frequency (maximum $100 \mathrm{~Hz}$ ), to enable the generation of required waveforms to the actuator while filtering correctly the PWM frequency from the inverter (30 kHz). The value $L_{s}$ is chosen to limit the current's variation through the diode. The components' parameters are presented in table I.

TABLE I

COMPONENTS' PARAMETER

\begin{tabular}{cccc}
\hline$L_{s}$ & $L_{b}$ & $C_{0}$ & $C$ \\
$10 \mathrm{mH}$ & $100 \mathrm{mH}$ & $4 \mathrm{mF}$ & $300 \mathrm{nF}$
\end{tabular}

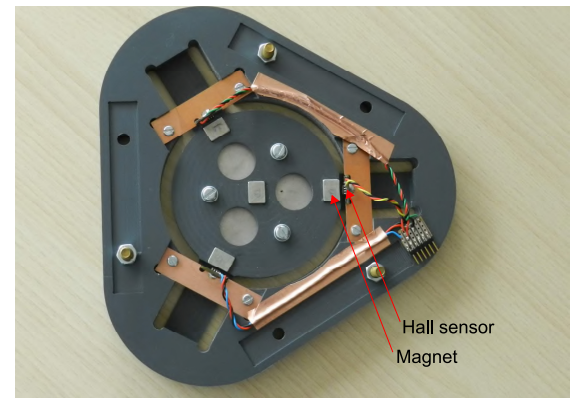

(a) Detail view of the compliant mechanism and sensors

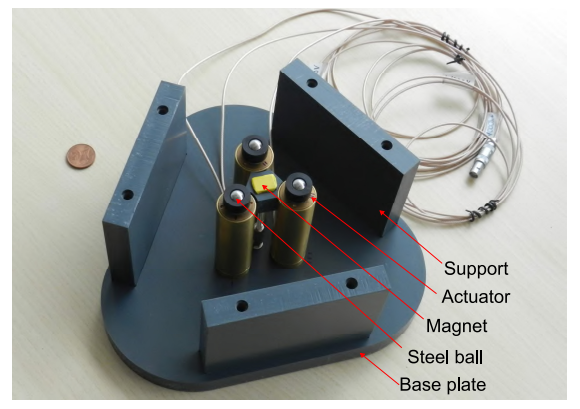

(b) Detail view of the actuators and the pre-load mechanism

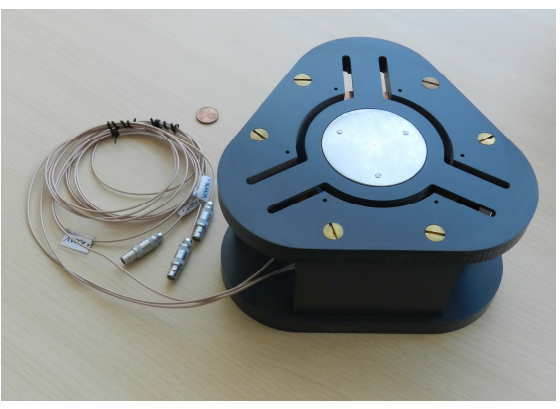

(c) Assembled prototype

Fig. 6. Prototype 
The complete electrical system is presented in Fig.8. An integrated DSP TMS $320 \mathrm{~F} 2812 \mathrm{~g}$ is used to generate the PWM duty cycle of three branches of inverter Semikron.

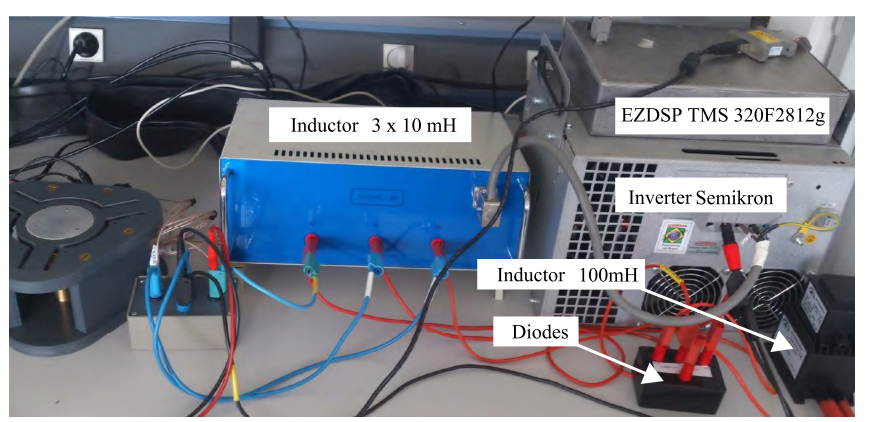

Fig. 8. Three-phase electrical system

\section{Modelling}

In order to have a model for process simulation and the control in a later study, a modelling of the complete system is performed by using the Energetic Macroscopic Representation (EMR). This tool helps construct a functional representation of systems in order to visualize the integral causality and power transmission between system's components by using a limited set of symbolic representations (see Appendix VII-A). Moreover, a control structure by the inversion-based principle can be directly deduced from this system's representation [17]. The EMR of complete system is established from the input of electrical source to the output of the mechanical system.

\section{A. EMR of electrical system}

The EMR of electrical system is directly built from the electrical diagram in Fig. 7. The inductors $L_{s}, L_{b}$ and the capacitors $C_{0}, C$ are modelled by energetic accumulators while the three-phase inverter is modelled in the ideal case by an electrical converter which is controlled by a duty cycle $m$. For a connection between the components $L_{s}, L_{b}$ and $C$, an electrical coupling is used. The complete EMR of electrical system is presented in Fig.9. It may be noted that the variable's vectors have a dimension of three, which represents the three-phase variables.

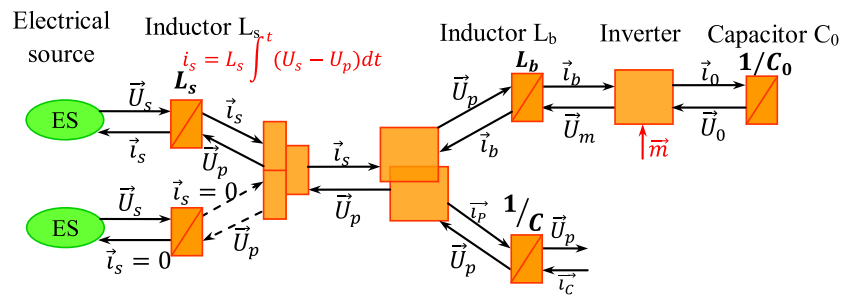

Fig. 9. EMR of three-phase electrical system

To represent the intermittent function of electrical system between the charging phase for the voltage of $C_{0}$ and the phase of vibrations' generation, a switch function is used to change the state of current $i_{s}$. From Fig.9, the states' interchange can be detected by the variation of voltage $U_{p}$. The phase of charging the voltage $U_{0}$ happens if and only if the voltage $U_{p}$ is less than to the value $U_{s}$, which corresponds to the period $i_{s}>0$.

\section{B. EMR of mechanical system}

1) EMR of mechanical coupling: Due to the small actuators' displacement, their velocities and forces are supposed to be always in the vertical direction as presented in Fig.10, where $v_{i}$ and $F_{i}$ are respectively the velocity and force found at the contacts. In Fig.10a, $\omega_{1}, \omega_{2}, \omega_{3}$ are represented for three instantaneous rotational vectors corresponding to three punctual contacts' velocities $v_{1}, v_{2}, v_{3}$.

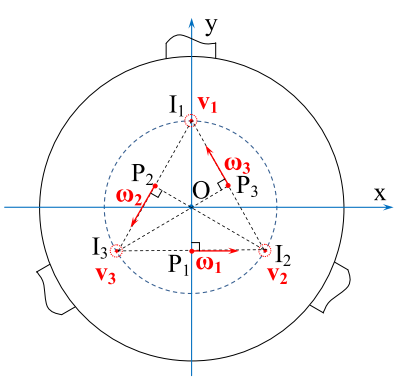

(a) Kinematic coupling

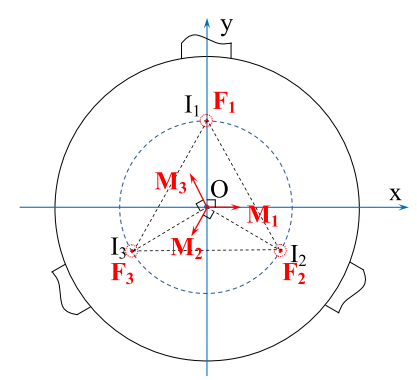

(b) Force coupling
Fig. 10. Schema for mechanical coupling's relation $\left(O I_{i}=R\right)$

The kinematic relation between the kinematic screw of the lower die at point $O\left\{\begin{array}{l}\overrightarrow{\omega_{O}} \\ \overrightarrow{v_{O}}\end{array}\right\}$ and the kinematic screw of each actuator at point $\mathrm{O}\left\{\frac{\overrightarrow{\omega_{i}}}{\vec{v}_{i / O}}\right\}$ is expressed as follows:

$$
\left\{\begin{array}{l}
\overrightarrow{\omega_{O}} \\
\overrightarrow{v_{O}}
\end{array}\right\}=\sum\left\{\begin{array}{l}
\overrightarrow{\omega_{i}} \\
\overrightarrow{v_{i / O}}
\end{array}\right\}=\left\{\begin{array}{c}
\sum \overrightarrow{\omega_{i}} \\
\sum \overrightarrow{O P_{i}} \times \overrightarrow{\omega_{i}}
\end{array}\right\}
$$

The relations of 2 rotations around Ox, Oy and displacement along $\mathrm{Oz}$ of the lower die with the actuators' velocities is obtained by projecting the equation (4) in the axes Ox, Oy, Oz.

$$
\mathbf{v}=\left[\begin{array}{l}
v_{1} \\
v_{2} \\
v_{3}
\end{array}\right]=\left[\begin{array}{ccc}
1 & R & 0 \\
1 & -\frac{R}{2} & -\frac{R \sqrt{3}}{2} \\
1 & -\frac{R}{2} & \frac{R \sqrt{3}}{2}
\end{array}\right]\left[\begin{array}{l}
v_{z} \\
\omega_{x} \\
\omega_{y}
\end{array}\right]=\mathbf{A} \mathbf{v}_{\mathbf{O}}
$$

Similarly, the wrench of the lower die at point $O\left\{\frac{\overrightarrow{F_{O}}}{M_{O}}\right\}$ is related to the wrench of each actuator at point $\mathrm{O}\left\{\frac{\overrightarrow{F_{i}}}{\overrightarrow{M_{i / O}}}\right\}$ by the following expression:

$$
\left\{\begin{array}{l}
\overrightarrow{F_{O}} \\
\overrightarrow{M_{O}}
\end{array}\right\}=\sum\left\{\begin{array}{c}
\vec{F}_{i} \\
\overrightarrow{M_{i / O}}
\end{array}\right\}=\left\{\begin{array}{c}
\sum \vec{F}_{i} \\
\sum \overrightarrow{O I_{i}} \times \vec{F}_{i}
\end{array}\right\}
$$

where $\vec{F}_{i}$ is the force generated by each actuator and $\vec{M}_{i}$ is the moment at point $\mathrm{O}$ by force $\vec{F}_{i}$. From (6), the relation of resultant force along $\mathrm{Oz}$ and moments around two axes $\mathrm{Ox}$ and $\mathrm{Oy}$ is presented by the following equation:

$$
\mathbf{F}_{\mathbf{O}}=\left[\begin{array}{c}
F_{z} \\
M_{x} \\
M_{y}
\end{array}\right]=\left[\begin{array}{ccc}
1 & 1 & 1 \\
R & -\frac{R}{2} & -\frac{R}{2} \\
0 & -\frac{R \sqrt{3}}{2} & \frac{R \sqrt{3}}{2}
\end{array}\right]\left[\begin{array}{l}
F_{1} \\
F_{2} \\
F_{3}
\end{array}\right]=\mathbf{B F}
$$

The input power $P_{\text {in }}=\mathbf{F}^{\top} \mathbf{v}$ is found equal to the output power $P_{\text {out }}=\mathbf{F}_{\mathbf{O}}{ }^{\top} \mathbf{v}_{\mathbf{O}}$, which demonstrates the power conservation within this model and the EMR can be applied to this mechanical coupling by representing it as a monophysic converter (see VII-A). 
2) EMR of compliant mechanism: With the small size and velocity of the designed mechanism, the effect of gravity, centrifugal and damping forces can be negligible in comparison with the forces generated by actuators (a few thousands Newtons). The dynamic equations of lower die is written in the following form:

$$
\mathbf{M}(\mathbf{q}) \ddot{\mathbf{q}}+\mathbf{K}(\mathbf{q}) \mathbf{q}=\mathbf{F}_{\mathbf{e}}
$$

where $\mathbf{M}, \mathbf{K}$ are respectively the inertia matrix and stiffness matrix of the compliant mechanism, $\mathbf{q}=\left[\begin{array}{llllll}x & y & z & \alpha_{x} & \alpha_{y} & \alpha_{z}\end{array}\right]$ is a vector of displacement and rotational angles along axis Ox, Oy, Oz. In the first approach, the friction force between lower die and workpiece is also neglected. The applied forces on the lower die are only the forces generated by the actuators and the forging process in the vertical direction. The vector $\mathbf{F}_{\mathbf{e}}$ can be expressed as follows : $\mathbf{F}_{\mathbf{e}}=$ $\left[\begin{array}{llllll}0 & 0 & F_{z}-F_{w p} & M_{x}-M_{w p_{X}} & M_{y}-M_{w p_{Y}} & 0\end{array}\right]^{T}$, where $F_{w p}, M_{w p_{X}}, M_{w p_{Y}}$ are the applied force and moments of workpiece to the lower die.

From the numerical values of inertia and stiffness matrix (see Appendix VII-B for the prototype), the displacement modes along $\mathrm{Ox}$ and $\mathrm{Oy}$, which are coupled with the corresponding rotations around $\mathrm{Oy}$ and $\mathrm{Ox}$, are found as the non-dominant modes of movement. The system can be reduced into a 3-degree-of-freedom system as follows:

$$
\left\{\begin{array}{l}
m_{x} \ddot{\alpha_{x}}+k_{x} \alpha_{x}=M_{x}-M_{w p_{X}} \\
m_{y} \ddot{\alpha_{y}}+k_{y} \alpha_{y}=M_{y}-M_{w p_{Y}} \\
m_{z} \ddot{z}+k_{z} z=F_{z}-F_{w p}
\end{array}\right.
$$

The EMR for the vertical movement's equation along the axis $\mathrm{Oz}$ is presented in Fig. 11.

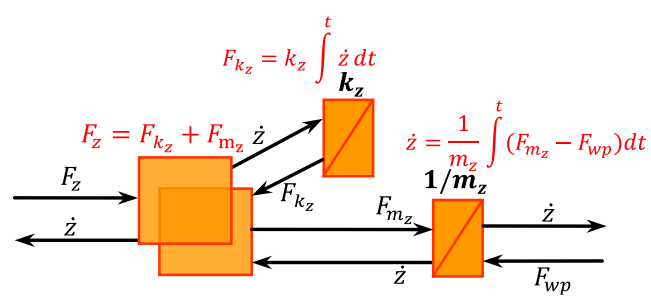

Fig. 11. EMR for displacement mode along $\mathrm{Oz}$

3) EMR of piezoelectric actuator: The EMR of piezoelectric actuator in quasi-static mode is developed in [4] to relate the input current $i_{p}$ and velocity $v_{p}$ with the output force $F_{p}$ and voltage $U_{p}$. In this model, the parameter $K_{c}$ presents the piezoelectric coefficient while $K_{s}$ is the actuator's stiffness. The piezoelectric actuator's model is connected to a contact which is represented by a mass $M_{c t}$ (dynamic mass of actuator and steel ball's mass) in series with a spring of the stiffness $K_{c t}$.

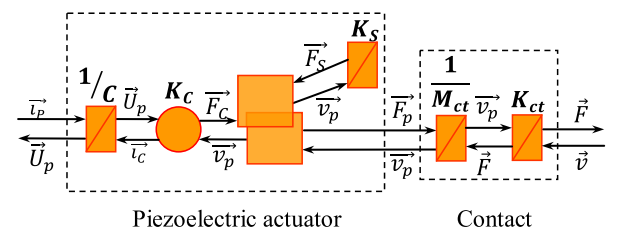

Fig. 12. EMR model for a piezoelectric actuator connected to a contact

The complete system's EMR is achieved by assembling the components' EMR, as presented in Fig.13, by connecting the same pairs of variables in each model.

\section{Experimental results and discussion}

To demonstrate the function in generating vibrations and validate the components' EMR of the complete system, this part presents the experimental results and compares with results obtained from the model.

1) Validation of electrical system's modelling: With the proposed mechanical configuration, the required waveforms of actuators' voltage for a progressive wave are a threephase sinusoidal voltage. This voltage is obtained by applying a three-phase sinusoidal duty cycle $\vec{m}$ to the DSP. The variation of duty cycle's mean value is used to trigger on and off the charging of capacitor $C_{0}$. This is done by setting the mean values such that the minimum voltage $U_{p}$ is above (phase of generating vibrations) or below (phase of charging) $U_{s}$, which is fixed to $100 \mathrm{~V}$ in the tests. The measured voltages of the two actuators and the corresponding currents $i_{s}$ are presented in Fig.14 in the case of generation a three-phase sinusoidal voltage with $50 \mathrm{~V}$ the amplitude. The current $i_{s}$ is greater than 0 every period for which the voltages $U_{p}$ are lower than $U_{s}=100 \mathrm{~V}$.

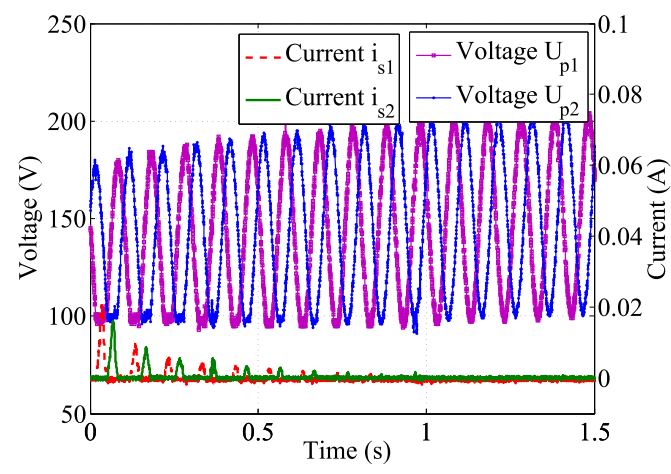

Fig. 14. Measured results in the transient phase of the three-phase voltage supply system

By applying the same duty cycle to the input of the electrical system's EMR, the simulated voltage $U_{p}$ and current $i_{s}$ are presented in Fig.15. The obtained results are qualitatively similar to the measure results. Simulated currents in the diodes follows the same trend as in the experiment. However, the actuator's average voltage increases because the diodes' conducting time is reduced due to the model's difference with the real inverter and the losses in the circuit. Nevertheless, this result shows a coherence of the model's behaviour to the real one.

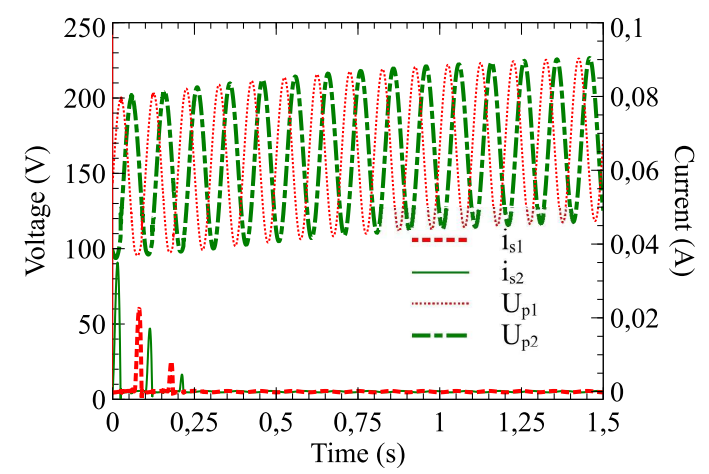

Fig. 15. Simulated results in the transient phase of the three-phase voltage supply system 


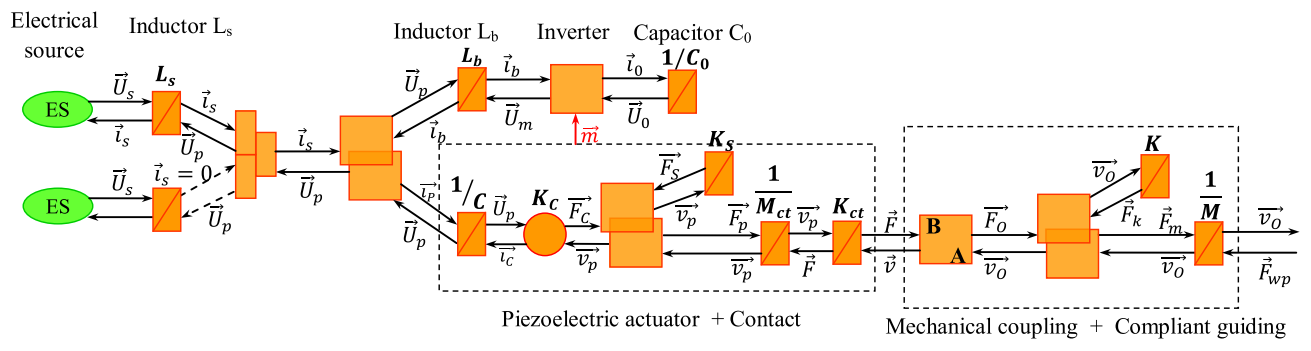

Fig. 13. EMR of the complete system

2) Validation of mechanical system's modelling: The mechanical system is tested in two experiments to validate its kinematics and the results are compared to one from simulation with the EMR. The first experiment is performed to generate a lower die's movement following a progressive wave. This movement results from two rotations around two axes, as demonstrated in the section III.A. This two rotations' speeds are difficult to measure directly. A laser vibrometer is then used to measure the displacement speeds along two axes of one screw fixed in the lower die's center (Fig.16).

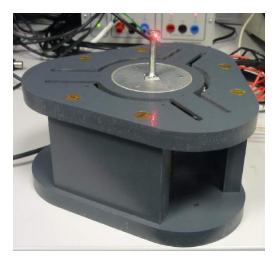

Fig. 16. Speed measurement by a laser vibrometer

In this test, a $50 \mathrm{~Hz}$ three-phase voltage with $100 \mathrm{~V}$ amplitude is applied to three actuators to generate a progressive wave in the lower die. The measured speed by vibrometer (Fig.17) confirms the presence of two rotations with the same amplitude and in quadrature. It also means that a progressive wave with the frequency $50 \mathrm{~Hz}$ is found on the lower die.
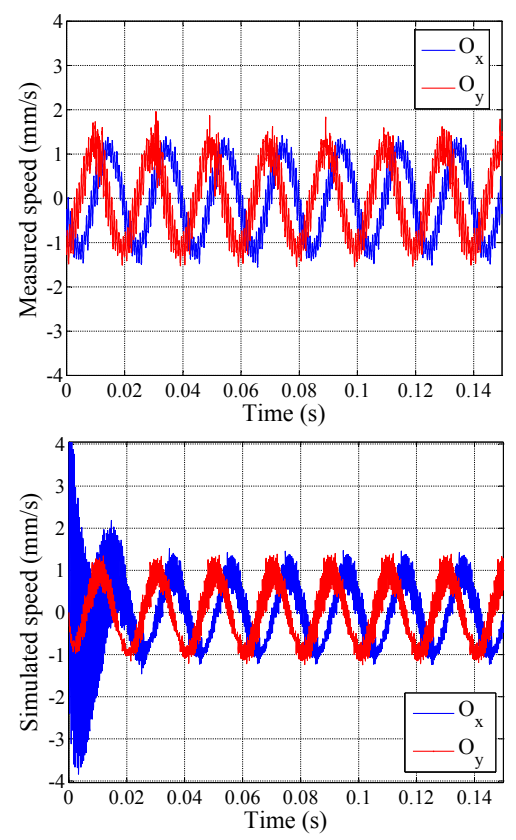

Fig. 17. Speed along Ox and Oy of a rod fixed in the lower die's center

The measurement of actuators' current is then used as the input $\overrightarrow{i_{p}}$ for the piezoelectric actuator's modelling in simulation. The output's rotation speed of mechanical system's model is used to estimate the speed of measured point by multiplying with the distance from the measure point (Fig.16) to the point $\mathrm{O}$ (about $39 \mathrm{~mm}$ ). With the established EMR, the simulated speed shows a good agreement with the measured values in phase and fairly well in amplitude. A transition phase is found in the simulated result because the measured input current $\overrightarrow{i_{p}}$ is not equal to zero at the beginning of simulation.

The second test is to generate a vertical triangular vibration by applying three $50 \mathrm{~Hz}$ voltages in triangular form presented in Fig.18. The actuators' currents are also measured and filtered to obtain the input as the previous test. The measured voltage and speed after filtering with the same filter are presented in Fig.18 in comparison with the simulated values. The two presented results show once again that the model gives a good approximation of the mechanical system's behaviour in the case of vertical movement.
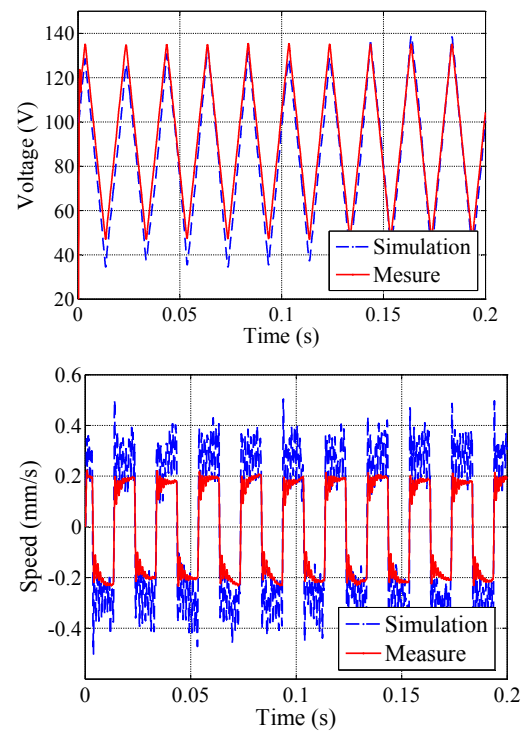

Fig. 18. Measured voltage and speed in comparison with simulated values

\section{Vi. Conclusion}

This paper presents a complete piezoelectric multiactuators system to assist a forging process. A compliant mechanism integrating three actuators is built to generate a progressive wave in combination with a vertical displacement on the lower die. A three-phase voltage supply system without transformer is also presented to supply frequency-variable high voltages to three actuators in order to achieve the desired movement. A modelling using EMR 
is established for both mechanical and electrical system to visualize the power transmission from the electrical input to the mechanical output, which gives us a global view for the control. The movement of a progressive wave and the vertical simulation is demonstrated by experimental validation and also confirmed by the simulation.

\section{APPENDIX}

A. EMR's basic elements

\begin{tabular}{|c|c|c|}
\hline Element & Symbol & Description \\
\hline Variable & $\underset{y}{\stackrel{x}{y}}$ & Action/ reaction variable $(x / y)$ \\
\hline Source & $\underset{E}{P}$ & Energetic source \\
\hline Accumulator & $\rightleftarrows / \rightleftarrows$ & Accumulator \\
\hline \multirow[t]{2}{*}{ Converters } & & Mono-physic converter \\
\hline & & Multi-physic converter \\
\hline \multirow[t]{2}{*}{ Couplings } & $=$ & Mono-physic coupling \\
\hline & $=\theta$ & Multi-physic coupling \\
\hline
\end{tabular}

\section{B. Inertia and stiffness matrix of prototype}

$$
\begin{gathered}
\mathbf{K}=10^{3}\left[\begin{array}{ccccccc}
5,14.10^{3} & 0 & 0 & 0 & -7,71 & 0 \\
0 & 5,14.10^{3} & 0 & 7,71 & 0 & 0 \\
0 & 0 & 49,77 & 0 & 0 & 0 \\
0 & 7,71 & 0 & 0,12 & 0 & 0 \\
-7,71 & 0 & 0 & 0 & 0,12 & 0 \\
0 & 0 & 0 & 0 & 0 & 5,68
\end{array}\right] \\
\mathbf{M}=10^{-3}\left[\begin{array}{cccccc}
94 & 0 & 0 & 0 & -0,39 & 0 \\
0 & 94 & 0 & 0,393 & 0 & 0 \\
0 & 0 & 94 & 0 & 0 & 0 \\
0 & 0,39 & 0 & 0,05 & 0 & 0 \\
-0,39 & 0 & 0 & 0 & 0,05 & 0 \\
0 & 0 & 0 & 0 & 0 & 0,09
\end{array}\right]
\end{gathered}
$$

\section{ACKNOWLEDGEMENT}

This work has been carried out within the framework of the Min Project of Inria, and the Stimtact Project of the Institut de Recherche sur les Composants Logiciels et Matriels pour la Communication Avance (IR CICA). Financial support for this study was provided by a grant from the European Union which invested in the Nord-Pas-de-Calais Region (FEDER funds) within the framework of the FUI TOUCHIT (Tactile Open Usage with Customized Haptic Interface Technology).

\section{REFERENCES}

[1] Z. Huang, M. Lucas, and M. J. Adams, "Influence of ultrasonics on upsetting of a model paste," Ultrasonics, vol. 40, pp. 43-48, 2002.

[2] D. Yusof, M. Lucas, and Z. Huang, "Superimposed ultrasonic oscillations in compression tests of aluminium," Ultrasonics, vol. 44, pp. e511-e515, 2006.

[3] R. Ly, C. Giraud-Audine, G. Abba, and R. Bigot, "Experimentally valided approach for the simulation of the forging process using mechanical vibration," International Journal on Material Forming, vol. 2, pp. 133-136, 2009.

[4] T. H. Nguyen, C. Giraud-Audine, B. Lemaire-Semail, G. Abba, and R. Bigot, "Modelling of piezoelectric actuators used in forging processes: principles and experimental validation," XXth International Conference on Electrical Machines (ICEM'2012), pp. 709-714, 2012.

[5] — , "Modelling of forging processes assisted by piezoelectric actuators : principles and experimental validation," IEEE Transactions on Industry Applications, vol. 50, no. 1, pp. 244-252, 2014.

[6] A. Khan, "Integration and optimization of vibration in metal forming process," Ph.D. dissertation, École Nationale Supérieure des Arts et Métiers, 2013.

[7] L. L. Howell, S. P. Magleby, and B. M. Olsen, Eds., Handbook of compliant mechanisms. Wiley, 2013.

[8] J. K. Salisbury, "Active stiffness control of a manipulator in cartesian coordinates," in 19th IEEE Conference on Decision and Control including the Symposium on Adaptive Processes, vol. 19, 121980 , pp. 95-100.

[9] J. Przemieniecki, Theory of matrix structural analysis. Dover Publications Inc., 1968.

[10] W. Khalil and E. Dombre, Modeling, Identification \& Control of Robots. Taylor \& Francis, 2002
[11] D. Wang, W. Zhu, Q. Yang, and W. M. Ding, "A high-voltage and high-power amplifier for driving piezoelectric stack actuators," Journal of Intelligent Material Systems and Structures, vol. 20, pp. 1987-2001, 2009.

[12] C. Wallenhauer, A. Kappel, B. Gottlieb, T. Schwebel, and T. Lüth, "Efficient class-b analog amplifier for a piezoelectric actuator drive," Mechatronics, vol. 19, no. 1, pp. 56 - 64, 2009.

[13] A. V. Carazo and K. Uchino, "Novel piezoelectric-based power supply for driving piezo actuators designed for active vibration damping applications," Journal of Electroceramics, vol. 7, pp. 197-201, 2001.

[14] R. Li, M. Loenneker, N. Froehleke, and J. Boecker, "Design of power supply for driving high power piezoelectric actuators,' in Industry Applications Society Annual Meeting, IAS '08. IEEE, 2008, pp. 1-6.

[15] D. H. Tran, J. F. Rouchon, B. Nogarede, and C. Viguier, "Design a power inverter and transformer for piezoelectric actuator," in 10th International Workshop on Electronics, Control, Measurement and Signals (ECMS), 2011, pp. 1-6.

[16] E. Goenaga, J.-P. Ferrieux, and J. Barbaroux, "Contactless power transfer system for high power piezoelectric actuators in aeronautical applications," in APEC Conference 2013, Long Beach, États-Unis, 2013.

[17] P. J. Barre, A. Bouscayrol, P. Delarue, E. Dumetz, F. Giraud, J. P. Hautier, X. Kestelyn, B. Lemaire-Semail, and E. Semail, "Inversion based control of electromechanical systems using causal graphical descriptions," IEEE-IECONO6 (Paris), nov 2006.

Thanh Hung Nguyen received his B.S. degree in Mechatronics from the Ho Chi Minh University of Technology, Vietnam, in 2006 and his M.S. degree from the École Nationale Supérieure de Mécanique et des Microtechniques de Besançon, France and the Hochschule Karlsruhe Technik und Wirtschaft, Germany in 2010. He is currently working towards the Ph.D degree at the École Nationale Supérieure d'Arts et Métiers, France. His Ph.D research focuses on design, modelling and control a system of multi-piezoelectric actuators to assist forging processes.

Christophe Giraud-Audine received his M.Eng. degree in mechanical engineering from the École Nationale Supérieure d'Arts et Métiers in 1992, and his Ph.D degree in electrical engineering from the Institut National Polytechnique de Toulouse in 1998. After two years spent as a Research Associate at the University of Sheffield, he joined an associate professor position at the École Nationale Supérieure d'Arts et Métiers. His current research focuses on the modelling and control of devices based on piezoelectric and shape memory alloys.

Michel Amberg has been teaching electronics at the University of Lille, France. He graduated from the École Normale Supérieure de Cachan, France in 1981. He has tutored more than a hundred bachelor's students during their projects in the field of telecommunications, computer science, and electronics. He is now a research engineer at IRCICA, and works on the electronic design of tactile devices.

Betty Lemaire-Semail received her $\mathrm{Ph}$. D degree in 1990 from the University of Paris XI, Orsay and habilitation degree in 1997 from the University of Lille 1. From 1990 to 1998, she was an assistant professor at the École Centrale of Lille and she is now a professor at the University of Lille 1 . She is a member of the electrical engineering and power electronics laboratory of Lille (L2EP) and head of the research axis related to control of electrical systems. She has studied electromagnetic motors and her main field of interest now deals with the modelling and control of piezoelectric actuators for positioning and force feedback applications.

Gabriel Abba has joined the "Ecole Normale Supérieure de Cachan", France, in 1979. He received the Doctorate degree in Electronics and Robotics from the University of Paris XI-Orsay, Paris, France, in 1986. Since 2004, he has been at The "Ecole National d'Ingénieurs de Metz", where he is currently a Professor of Electrical and Computer Engineering. His research interests include development, modeling and control of robots, specially control of legged robots, visual servoing, manipulator control, design of robots and modelling and control of electromechanical actuators for industrial applications. He has made many contributions to robot dynamics and legged locomotion, and he has over 110 publications in Journals or Conferences.

Régis Bigot received his M.Eng. degree in mechanical engineering from the École Nationale Supérieure d'Arts et Métiers in 1992. In 1993 he obtained a master of sciences in Physics and Chemistry of Materials of University of Lille and his Ph.D degree in physical and metallurgy from the University of Lille in January 1996. After three years as technical teacher at Lille and Metz, he joined an associate professor position at the École Nationale Supérieure d'Arts et Métiers in September 1998. In September 2008, he becomes full professor in same institute. Since October 2011, he is director of Design, Manufacturing Engineering and Control Laboratory. His current research focuses on the manufacturing process (bulk forming, thixoforming, assembly, ...), integrated product and process design and CAPP. 